\title{
Long-Term Follow-Up of Contrast-Induced Acute Kidney Injury: A Study from a Developing Country
}

\author{
Ashraf O. Oweis ${ }^{1 D},{ }^{1}$ Sameeha A. Alshelleh, ${ }^{2}$ Nesreen Saadeh, ${ }^{3}$ Mohamad I. Jarrah, \\ Rasheed Ibdah, ${ }^{4}$ and Karem H. Alzoubi ${ }^{5}{ }^{5}$ \\ ${ }^{1}$ Division of Nephrology, Department of Medicine, Jordan University of Science and Technology, Irbid, Jordan \\ ${ }^{2}$ Division of Nephrology, Department of Medicine, The University of Jordan, Amman, Jordan \\ ${ }^{3}$ Department of Medicine, Jordan University of Science and Technology, Irbid, Jordan \\ ${ }^{4}$ Division of Cardiology, Department of Medicine, Jordan University of Science and Technology, Irbid, Jordan \\ ${ }^{5}$ Department of Clinical Pharmacy, Jordan University of Science and Technology, Irbid, Jordan
}

Correspondence should be addressed to Ashraf O. Oweis; ashrafowais78@gmail.com

Received 24 July 2020; Revised 4 December 2020; Accepted 13 December 2020; Published 21 December 2020

Academic Editor: Antonio Bozzani

Copyright ( 92020 Ashraf O. Oweis et al. This is an open access article distributed under the Creative Commons Attribution License, which permits unrestricted use, distribution, and reproduction in any medium, provided the original work is properly cited.

\begin{abstract}
Introduction. Contrast-induced acute kidney injury (CI-AKI) is a worldwide known complication related to the use of contrast media with either imaging or angiography; it carries its own complications and effect on both morbidity and mortality; early identification of patients at risk and addressing modifiable risk factors may help reducing risk for this disease and its complications. Methods. This was a prospective observational study, where all patients admitted for cardiac catheterization between June 2015 and January 2016 were evaluated for CI-AKI. There were two study groups: contrast-induced acute kidney injury (CI-AKI) group, and noncontrast-induced acute kidney injury (non-CI-AKI) group. Results. Patients $(n=202)$ were included and followed up for 4 years. Death and development of chronic kidney disease (CKD) need for another revascularization were the end points. The incidence of CI-AKI was $14.8 \%$.In univariate analysis, age $(P=0.016)$ and serum albumin at admission $(P=0.001)$ were statistically significant predictors of overall death. Age $(P=0.002), \mathrm{HTN}(P=0.002)$, DM $(P=0.02)$, and the use of diuretics $(P=0.001)$ had a statistically significant impact on eGFR. The rate of recatheterization was not statistically significant between the two groups (61 (35.5\%) for the non-CI-AKI vs. 12 (40\%) for the other group; $P=0.63$ ). Some inflammatory markers (NGAL $P=0.06$, IL-19 $P=0.08$ ) and serum albumin at admission $P=0.07$ had a trend toward a statistically significant impact on recatheterization. Death $(P=0.66)$ and need for recatheterization $(P=0.63)$ were not statistically different between the 2 groups, while the rate of eGFR decline in for the CI-AKI was significant $(P=0.004)$. Conclusion. CI-AKI is a common complication post percutaneous catheterization (PCI), which may increase the risk for CKD, but not death or the need for recatheterization. Preventive measures must be taken early to decrease the morbidity.
\end{abstract}

\section{Introduction}

Contrast-induced acute kidney injury (CI-AKI) is a potentially preventable and reversible cause of acute kidney injury (AKI). Multiple factors are associated with the development of CI-AKI, some are modifiable such as drugs, type, and amount of contrast media used, and others are not such as preexisting chronic kidney disease (CKD) or heart failure [1].

Though it is usually a reversible condition, there is increasing evidence of adverse long-term outcome including increased morbidity and mortality [2]. Identifying risks and adapting some protocols may help decreasing the incidence and impact of this disease $[3,4]$. It is apparent that CI-AKI contributes to long-term mortality, revascularization need, and increased risk for CKD $[4,5]$.

The incidence of CI-AKI has been variable among studies (2-15\%), and the definition to be used for CI-AKI is still debatable, whether to use AKIN, RIFLE with MDRD, or RIFLE with CKD-EPI or CK or KIDOGI is also variable between studies [6]. According to a large cohort of patients who underwent PCI post-STEMI, the RIFLE with CKD-EPI was the most to determine and predict complications like 
inhospital mortality, one-year mortality, and need for dialysis at 1 year [7]. In general, the incidence of CI-AKI post-PCI can differ even on the same cohort according to the definition used for CI-AKI [8]. The aim of this study is to evaluate the long-term outcome of CI-AKI on mortality, the rate of recatheterization, and the development of $\mathrm{CKD}$ in a health setting of a developing country.

\section{Method}

This study was a prospective observational study. All patients admitted for cardiac catheterization of King Abdulla University Hospital, Irbid, Jordan, over a period of 6 months were included. This tertiary hospital is the largest hospital at north province of Jordan with over 700 beds capacity. This study had 326 patients as participants and represents a secondary analysis of our previous study [9]. Every participant had to sign the consent form. For the purpose of measuring creatinine levels, blood was withdrawn 48 hours after the procedure.

The CI-AKI was defined as $>25 \%$ or $44 \mathrm{mmol} / \mathrm{l}$ increase in serum creatinine from the baseline level by 48-72 hours-without any other obvious cause-after administration of contrast media [10-12]. The Modification of Diet in Renal Disease (MDRD) equation was used to estimate glomerular filtration rate (eGFR). Low-osmolality contrast media (CM) was the only used media among patients in the current study (Lopamidol, Bayer, Germany, $616 \mathrm{mOsm} / \mathrm{kg} \mathrm{H}_{2} \mathrm{O}$ ). Only 202 patients of the original 326 patients included had a second sample withdrawn, thus, were qualified to remain in the study. There were two study groups: the contrast-induced acute kidney injury (CI-AKI) group and noncontrastinduced acute kidney injury (non-CI-AKI) group. Patients were followed for 4 years; data for long-term follow-up was obtained either from the hospital records or phone calls. The primary endpoints were mortality, development of $\mathrm{CKD}$, and the need for recatheterization by the end of follow-up. This study protocol was approved by the institutional review board of Jordan University of Science and Technology. Written informed consent forms were obtained from all study subjects.

2.1. Cytokines Measurements. Serum samples were stored at $-80^{\circ} \mathrm{C}$ in aliquots until the time of analysis. The levels of IL-1 $\alpha$, IL-6, IL-19, IL-20, IL-21, IL-22, and IL-33 were directly measured-without dilution-from serum, whereas the levels of NGAL were done after 1:20 dilution. Measurement was carried out according to commercial kits as per the manufacturers' instructions (IL- $1 \alpha$ (R\&D Systems, Inc., Minneapolis, MN, USA), IL-6 (R\&D Systems), IL-19 (R\&D Systems), IL-20 (R\&D Systems), IL-21 (eBioscience, San Diego, CA, USA), IL-22 (R\&D Systems), IL-33 (R\&D Systems), and NGAL (RayBio, Norcross, GA, USA). The samples in reaction mixtures were read at $450 \mathrm{~nm}$ wavelength.

2.2. Statistical Analysis. Analysis was performed using STATA/MP, version 14.0 (StataCorp LLC, College Station, TX, US). Data were described using percentages for categorical variables and means and SD for continuous variables.
There were two study groups in the current study: patients with CI-AKI and those without CI-AKI. To compare the means in continuous variables, $t$-test for independent samples was performed. Chi-squared test was used to test the association between the demographic, clinical, and other relevant characteristics of the participants and the incidence rates of CI-AKI (with and without $\mathrm{CI}-\mathrm{AKI}$ ). To determine the influence of factors associated with CI-AKI, multivariate binary logistic regression was done.

\section{Results}

The incidence of CI-AKI was $14.8 \%$ (30 patients). Patients' age was $55.8 \pm 9.7$ years, $28.2 \%$ of them were females, and $54.2 \%$ of them were either current or past smokers. Additionally, $43.6 \%$ of the patients' diabetes mellitus, $68.8 \%$ had hypertension, $26.8 \%$ had congestive heart failure, $10.4 \%$ had cerebrovascular accident, and $47.0 \%$ had coronary artery disease. At the end of follow-up, a total of 10 patients died, nine in the non-CI-AKI group vs. one in the CI-AKI group $(P=0.66)$; the mean time to death was 22.1 months $(\mathrm{SD} \pm 14.1$; Figure 1$)$. In univariate analysis, age $(P=0.016)$ and serum albumin at admission $(P=0.001)$ were statistically significant predictor of overall death, while patients' gender, underlying comorbidities, drugs, and different inflammatory markers (IL-1, IL-6, IL-19, IL-20, Il-21, IL-22, IL-33, and CRP) did not have an impact on mortality. Although the differences between the mean eGFRs, by the end of the follow-up period, were not statistically significant $(85.4 \mathrm{ml} / \mathrm{min}$ for the CI-AKI vs. $79.2 \mathrm{ml} / \mathrm{min}$ for the other group $(P=0.31))$, decline in eGFR for the CI-AKI was significant when pre- versus post-eGFRs were compared. A drop from $105.4 \mathrm{ml} / \mathrm{min}$ to $85.4 \mathrm{ml} / \mathrm{min}$ vs. $85.2 \mathrm{ml} / \mathrm{min}$ to $79.2 \mathrm{ml} / \mathrm{min}, P=0.004$; Figure 2 . Age $(P=0.002)$ but not gender $(P=0.34)$ was associated with worsening eGFR. HTN $(P=0.002)$, DM $(P=0.02)$, and the use of diuretics $(P=0.001)$ had a statistically significant impact on eGFR.

Mean time to recatheterization was 17.5 months $(\mathrm{SD} \pm 12.5)$; 15.8 months for the CI-AKI group (SD \pm 13.9$)$ vs. 17.8 months for the other group ( $\mathrm{SD} \pm 12.3), P=0.61$. The rate of recatheterization was not statistically significant between the two groups (61 (35.5\%) for the non-CI-AKI vs. $12(40 \%)$ for the other group; $P=0.63)$. As expected, history of CAD $(P=0.001)$ and the use of statins $(P=0.004)$ had an effect on the rate of recatheterization, while age, gender, comorbidities, and drugs did not. Patients with more than one stent inserted were more likely to undergo recatheterization $(P=0.003)$. Some inflammatory markers (NGAL- $P=0.06$, IL19 $-P=0.08)$ and serum albumin at admission $(P=0.07)$ had a trend toward statistically significant impact on recatheterization, see Table 1.

\section{Discussion}

In the current study cohort, the incidence for CI-AKI was $14.8 \%$, which was close to the results of the cohort by Uzunhasan et al., where CI-AKI happened in $16.4 \%$ of patients who underwent catheterization for ACS [5]. Having one or multiple vessels are catheterized during the PCI 


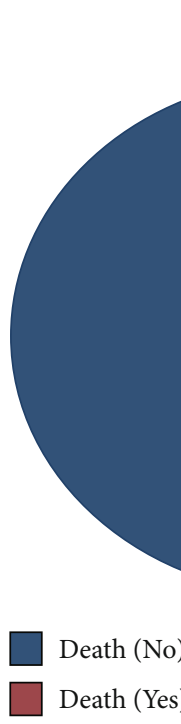

CI-AKI (-)

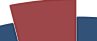

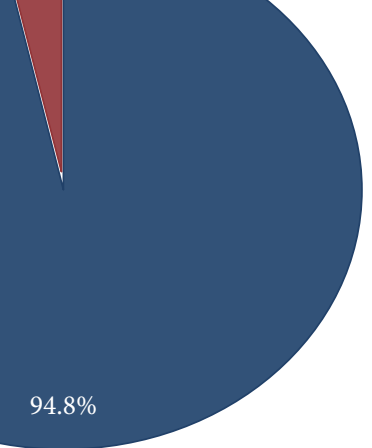

$5.2 \%$

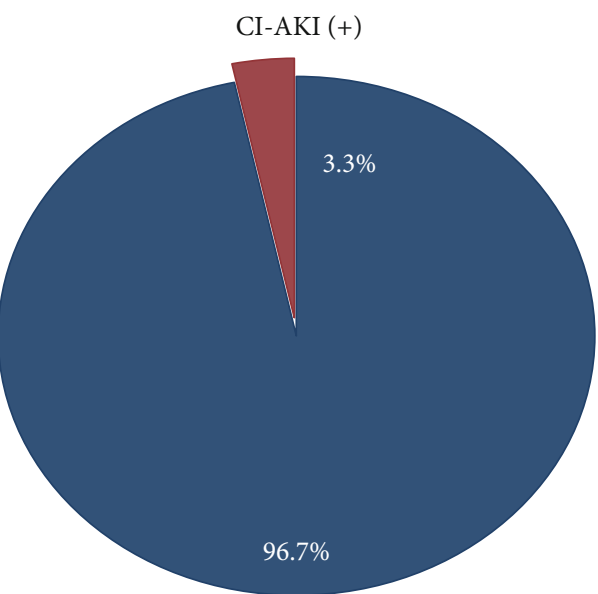

FIgURE 1: Mortality based on CI-AKI status.

CI-AKI (-)

CI-AKI (+)

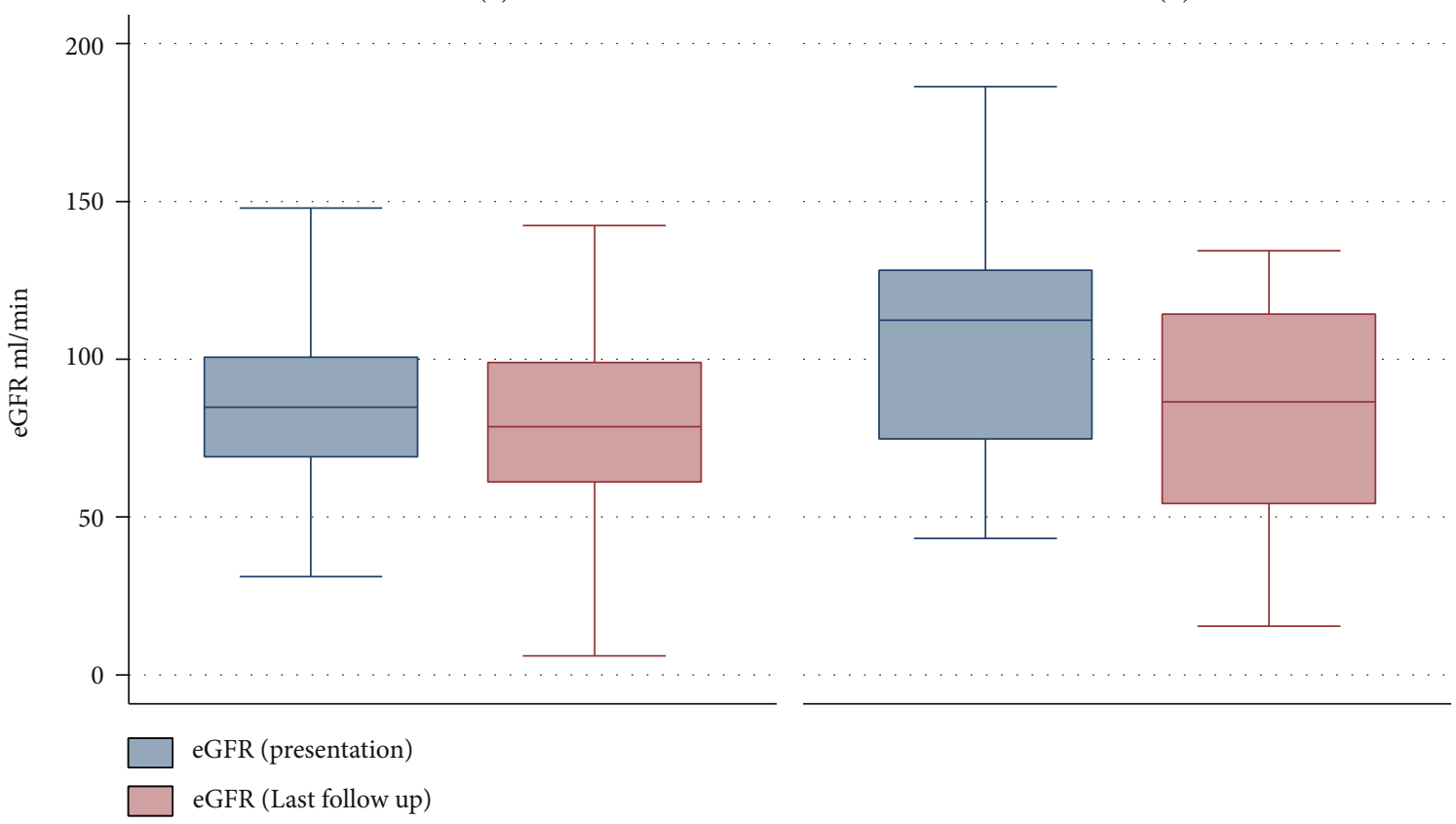

Figure 2: eGFR by CI-AKI status.

postcardiac event no effect on incidence of CI-AKI $[13,14]$. Predictors for CI-AKI were as follows: age, HTN, DM, and the use of diuretics. As expected, with aging, multiple factors can affect negatively influence kidneys and decrease eGFR such as increase in oxidative stress, decrease in local nitric oxide, decreased renal plasma flow, atherosclerotic changes, and damage to filtration barriers. A recent meta-analysis review done by Morcos et al. showed age as risk for CI-AKI; factors like hypertension, DM, contrast volume, and anemia will increase risk too [1]. Thrombolysis in myocardial infarction (TIMI) Risk Index (TRI) was found to be as independent risk factor for CI-AKI in patients who underwent PCI for ACS [15].
Serum albumin precatheterization can increase the risk for CI-AKI due to its relationship with endothelial dysfunction and background inflammatory response. This was shown in a cohort by Murat et al. in patients who underwent PCI for ACS, where serum albumin level was also a risk for CI-AKI [16]. Prealbumin levels can be associated with increased risk for CI-AKI and long-term mortality in elderly undergoing PCI [17].

Other biomarkers like NGAL are noticed to increase with CI-AKI especially with advanced stages of CKD urinary NGAL can predict the risk and severity of CI-AKI $[18,19]$. Still, some studies did not show a significant predictive value 
TABLE 1: Effect of different markers on outcomes.

\begin{tabular}{lccc}
\hline Marker & Death $(P$ value $)$ & CKD $(P$ value $)$ & $\begin{array}{c}\text { Recatheterization } \\
(P \text { value })\end{array}$ \\
\hline IL1 & $\mathbf{0 . 0 3}$ & 0.38 & 0.39 \\
IL6 & 0.41 & 0.18 & 0.30 \\
IL19 & 0.47 & 0.25 & $\mathbf{0 . 0 8}$ \\
IL20 & 0.93 & 0.85 & 0.85 \\
IL21 & 0.33 & 0.68 & 0.22 \\
IL22 & 0.79 & 0.50 & 0.40 \\
IL33 & 0.91 & 0.34 & 0.80 \\
NGAL & 0.19 & 0.65 & $\mathbf{0 . 0 6}$ \\
CRP & 0.93 & 0.20 & 0.28 \\
S. albumin & $\mathbf{0 . 0 0 1}$ & 0.15 & $\mathbf{0 . 0 7}$ \\
Hb & 0.15 & $\mathbf{0 . 0 6}$ & 0.37 \\
Vitamin D & 0.87 & 0.67 & 0.94 \\
\hline
\end{tabular}

of serum NGAL that allows prediction of CI-AKI in patients undergoing PCI post-ST elevation myocardial infarction (STEMI) [20]. Other markers in urine and blood were also studied in trial to predict and diagnose CI-AKI early enough to reduce complications [21].

The CI-AKI did not affect mortality in the current cohort. The incidence for mortality was $3.3 \%$ in CI-AKI group vs. $5.2 \%$ in non-CI-AKI group with mean time to death 22.1 months ( $S D \pm 14.1)$. At the same time, the difference between these two groups in the degree of eGFR drop or change was not statistically significant. These findings regarding mortality and change in renal function correlate well with findings from Ribitsch et al. cohort where no difference in creatinine was noticed or mortality by 2 years follow-up [2].

Baseline kidney function, being a CKD patient, may not affect the risk to develop CI-AKI but has its effect on both inhospital and long-term mortality post PCI. Even risk for future cardiovascular adverse events like STMI, NSTEMI, and stroke were higher in patients with CI-AKI $[22,23]$. Worse long-term outcomes were noticed in patients who underwent PCI for left main coronary artery stenosis with cardiogenic shock too [24]. In general, the length of hospital stay specifically decreased with adapting PCI if compared with patients with ACS and no PCI, risk for longer hospital stay were older age, female gender, and pacific ethnicity in addition to no PCI or need for CABG [25]. Short-term post-PCI complications such as leading to hospitalization and increased ninety days mortality are expected to be higher in patients with incomplete revascularization and with TIMI flow $<3$ at end of PCI especially for the left main coronary artery [26].

Regarding the need for another catheterization for revascularization, nine RCTs were studied by Xu et al. showed that complete revascularization if compared with infarct-related coronary artery revascularization only was associated with lower cardiac complications such as lower rate of revascularization, lower cardiac events, lower cardiac death, and allcause mortality. It was also worth to mention that rate of CI-AKI was not different between both groups [27]. Others also showed superiority of complete revascularization over infarct-related coronary artery revascularization [28].

In CKD patients, the risk for incomplete revascularization is higher and as expected is associated with higher complications and worse outcomes [29]. In some studies, the tendency of recatheterization for revascularization is lower in CKD patients if compared with others [30,31].

We found that comorbidities did not affect the rate for recatheterization for revascularization in our cohort, which is relatively small. Still, being a patient with diabetes will have a constant risk for ACS and its complications and fatality if compared with others, which their risks decreased over time with introduction of revascularization procedures [32]. Loading dose of statins prerevascularization was found to be associated with lower risk of CI-AKI and complications $[33,34]$. In our cohort, the use of statins was also associated with lower rate for events and revascularization.

We evaluated additional factors like serum interleukins; we found that IL-1 has an effect on mortality as outcome but not revascularization. IL-1 may initiate an inflammatory response and increase atherosclerosis especially in CKD patients [35]. The IL-19 may play some role in the rate of revascularization. This may be due to the effect of IL-19 effect on enhancing angiogenesis by macrophage polarization and decrease risk for atherosclerosis [36].

In conclusion, CI-AKI is a significant contributor to both short-term and long-term complications post-PCI. Efforts must be taken to identify patients at risk for CI-AKI, which may decrease cardiac morbidity and mortality, risk for $\mathrm{CKD}$, and rate of revascularization.

\section{Data Availability}

Data will be available upon request via e-mailing the corresponding author.

\section{Conflicts of Interest}

No potential conflict of interest was reported by the authors.

\section{Acknowledgments}

This study was funded by the Jordan University of Science and Technology.

\section{References}

[1] R. Morcos, M. Kucharik, P. Bansal et al., "Contrast-induced acute kidney injury: review and practical update," Clinical Medicine Insights Cardiology, vol. 13, article 1179546819878680 , 2019.

[2] W. Ribitsch, J. H. Horina, F. Quehenberger, A. R. Rosenkranz, and G. Schilcher, "Contrast induced acute kidney injury and its impact on mid-term kidney function, cardiovascular events and mortality," Scientific Reports, vol. 9, no. 1, p. 16896, 2019.

[3] M. Almendarez, H. S. Gurm, J. Mariani Jr. et al., "Procedural strategies to reduce the incidence of contrast-induced acute kidney injury during percutaneous coronary intervention," JACC Cardiovascular interventions, vol. 12, no. 19, pp. 18771888, 2019. 
[4] C. Vergadis, G. Festas, E. Spathi, P. Pappas, and S. Spiliopoulos, "Methods for reducing contrast use and avoiding acute kidney injury during endovascular procedures," Current Pharmaceutical Design, vol. 25, no. 44, pp. 4648-4655, 2019.

[5] I. Uzunhasan, A. Yildiz, S. Arslan et al., "Contrast-induced acute kidney injury is associated with long-term adverse events in patients with acute coronary syndrome," Angiology, vol. 68, no. 7, pp. 621-626, 2017.

[6] V. Pistolesi, G. Regolisti, S. Morabito et al., "Contrast medium induced acute kidney injury: a narrative review," Journal of Nephrology, vol. 31, no. 6, pp. 797-812, 2018.

[7] J. Silvain, L. S. Nguyen, V. Spagnoli et al., "Contrast-induced acute kidney injury and mortality in ST elevation myocardial infarction treated with primary percutaneous coronary intervention," Heart (British Cardiac Society), vol. 104, no. 9, pp. 767-772, 2018.

[8] B. Guillon, F. Ecarnot, C. Marcucci et al., "Incidence, predictors, and impact on six-month mortality of three different definitions of contrast-induced acute kidney injury after coronary angiography," The American Journal of Cardiology, vol. 121, no. 7, pp. 818-824, 2018.

[9] A. O. Oweis, S. A. Alshelleh, A. K. Daoud, M. M. Smadi, and K. H. Alzoubi, "Inflammatory milieu in contrast-induced nephropathy: a prospective single-center study," Int J Nephrol Renovasc Dis, vol. Volume 11, pp. 211-215, 2018.

[10] O. Caspi, M. Habib, Y. Cohen et al., “Acute kidney injury after primary angioplasty: is contrast-induced nephropathy the culprit?," Journal of the American Heart Association, vol. 6, no. 6, 2017.

[11] I. Goldenberg and S. Matetzky, "Nephropathy induced by contrast media: pathogenesis, risk factors and preventive strategies," CMAJ, vol. 172, no. 11, pp. 1461-1471, 2005.

[12] J. Schilp, C. de Blok, M. Langelaan, P. Spreeuwenberg, and C. Wagner, "Guideline adherence for identification and hydration of high-risk hospital patients for contrastinduced nephropathy," BMC Nephrology, vol. 15, no. 1, p. 2, 2014.

[13] S. Chatterjee, A. Kundu, D. Mukherjee et al., "Risk of contrastinduced acute kidney injury in ST-elevation myocardial infarction patients undergoing multi-vessel intervention-metaanalysis of randomized trials and risk prediction modeling study using observational data," Catheterization and Cardiovascular Interventions: Official Journal of the Society for Cardiac Angiography \& Interventions, vol. 90, no. 2, pp. 205212, 2017.

[14] B. N. Mukete, R. A. Riehl, A. Alonso, R. Samson, A. Jaiswal, and T. H. Le Jemtel, "Multivessel revascularization does not increase contrast-induced acute kidney injury incidence in acute myocardial infarction: a meta-analysis," American Journal of Cardiovascular Drugs: Drugs, Devices, and Other Interventions, vol. 16, no. 6, pp. 419-426, 2016.

[15] T. Cinar, Y. Karabag, V. Ozan Tanik, M. Cagdas, I. Rencuzogullari, and A. Oz, "The investigation of TIMI risk index for prediction of contrast-induced acute kidney injury in patients with ST elevation myocardial infarction," Acta Cardiologica, vol. 75, no. 1, pp. 77-84, 2020.

[16] S. N. Murat, A. Kurtul, and M. Yarlioglues, "Impact of serum albumin levels on contrast-induced acute kidney injury in patients with acute coronary syndromes treated with percutaneous coronary intervention," Angiology, vol. 66, pp. 732-737, 2014.
[17] Z. B. You, K. Y. Lin, W. P. Zheng et al., “Association of prealbumin levels with contrast-induced acute kidney injury in elderly patients with elective percutaneous coronary intervention," Clinical Interventions in Aging, vol. Volume 13, pp. 641649, 2018.

[18] K. Akrawinthawong, J. Ricci, L. Cannon et al., "Subclinical and clinical contrast-induced acute kidney injury: data from a novel blood marker for determining the risk of developing contrast-induced nephropathy (ENCINO), a prospective study," Renal Failure, vol. 37, pp. 187-191, 2014.

[19] A. Tasanarong, P. Hutayanon, and D. Piyayotai, "Urinary neutrophil gelatinase-associated lipocalin predicts the severity of contrast-induced acute kidney injury in chronic kidney disease patients undergoing elective coronary procedures," $B M C$ Nephrology, vol. 14, no. 1, p. 270, 2013.

[20] L. S. Nguyen, V. Spagnoli, M. Kerneis et al., "Evaluation of neutrophil gelatinase-associated lipocalin and cystatin $\mathrm{C}$ as biomarkers of acute kidney injury after ST-segment elevation myocardial infarction treated by percutaneous coronary intervention," Archives of Cardiovascular Diseases, vol. 112, no. 3, pp. 180-186, 2019.

[21] C. R. Parikh, C. Liu, M. K. Mor et al., "Kidney biomarkers of injury and repair as predictors of contrast-associated AKI: a substudy of the PRESERVE trial," American Journal of Kidney Diseases: The Official Journal of the National Kidney Foundation, vol. 75, no. 2, pp. 187-194, 2020.

[22] M. Maioli, A. Toso, M. Leoncini, M. Gallopin, N. Musilli, and F. Bellandi, "Persistent renal damage after contrast-induced acute kidney injury: incidence, evolution, risk factors, and prognosis," Circulation, vol. 125, no. 25, pp. 3099-3107, 2012.

[23] J. A. Neyra, S. Shah, R. Mooney, G. Jacobsen, J. Yee, and J. E. Novak, "Contrast-induced acute kidney injury following coronary angiography: a cohort study of hospitalized patients with or without chronic kidney disease," Nephrology, dialysis, transplantation: official publication of the European Dialysis and Transplant Association-European Renal Association, vol. 28, no. 6, pp. 1463-1471, 2013.

[24] J. Yeoh, N. Andrianopoulos, C. M. Reid et al., "Long-term outcomes following percutaneous coronary intervention to an unprotected left main coronary artery in cardiogenic shock," International journal of cardiology., vol. 308, pp. 20-25, 2020.

[25] T. K. M. Wang, C. Grey, Y. Jiang, R. Jackson, and A. Kerr, "Trends in length of stay following acute coronary syndrome hospitalisation in New Zealand 2006-2016: ANZACS-QI 32 study," The New Zealand Medical Journal, vol. 133, no. 1508, pp. 29-42, 2020.

[26] A. F. Castro-Mejia, M. E. Ortega-Armas, H. Mejia-Renteria et al., "Short-term clinical outcomes of percutaneous coronary intervention of unprotected left main coronary disease in cardiogenic shock," Catheterization and Cardiovascular Interventions: Official Journal of the Society for Cardiac Angiography \& Interventions, vol. 95, pp. 515-521, 2019.

[27] H. Xu, X. Zhang, J. Li, H. Liu, X. Hu, and J. Yang, "Complete versus culprit-only revascularization in patients with STsegment elevation myocardial infarction and multivessel disease: a meta-analysis of randomized trials," BMC Cardiovascular Disorders, vol. 19, no. 1, p. 91, 2019.

[28] V. Pasceri, G. Patti, F. Pelliccia et al., "Complete revascularization during primary percutaneous coronary intervention reduces death and myocardial infarction in patients with multivessel disease: meta-analysis and meta-regression of randomized trials," 
JACC Cardiovascular interventions, vol. 11, no. 9, pp. 833-843, 2018.

[29] T. Cardi, A. Kayali, A. Trimaille et al., "Prognostic value of incomplete revascularization after percutaneous coronary intervention following acute coronary syndrome: focus on CKD patients," Journal of Clinical Medicine, vol. 8, no. 6, p. 810, 2019 .

[30] G. Chernin, S. Chen, O. Ozan et al., "Percutaneous coronary intervention does not lower cardiovascular outcomes in patients with chronic kidney disease," American Journal of Nephrology, vol. 50, no. 6, pp. 465-472, 2019.

[31] S. Kotwal, I. Ranasinghe, D. Brieger, P. A. Clayton, A. Cass, and M. Gallagher, "The influence of chronic kidney disease and age on revascularization rates and outcomes in acute myocardial infarction - a cohort study," European Heart Journal Acute Cardiovascular Care, vol. 6, pp. 291-298, 2017.

[32] S. H. Read, C. M. Fischbacher, H. M. Colhoun et al., "Trends in incidence and case fatality of acute myocardial infarction, angina and coronary revascularisation in people with and without type 2 diabetes in Scotland between 2006 and 2015," Diabetologia, vol. 62, no. 3, pp. 418-425, 2019.

[33] J. Zhang, Y. Guo, Q. Jin, L. Bian, and P. Lin, "Meta-analysis of rosuvastatin efficacy in prevention of contrast-induced acute kidney injury," Drug Design, Development and Therapy, vol. 12, pp. 3685-3690, 2018.

[34] X. Zhou, J. Dai, X. Xu et al., "Comparative efficacy of statins for prevention of contrast-induced acute kidney injury in patients with chronic kidney disease: a network meta-analysis," Angiology, vol. 70, no. 4, pp. 305-316, 2019.

[35] P. M. Ridker, J. G. Mac Fadyen, R. J. Glynn et al., "Inhibition of interleukin $-1 \beta$ by canakinumab and cardiovascular outcomes in patients with chronic kidney disease," Journal of the American College of Cardiology, vol. 71, no. 21, pp. 2405-2414, 2018.

[36] K. Gabunia and M. V. Autieri, "Interleukin-19 can enhance angiogenesis by Macrophage Polarization," Macrophage, vol. 2, article e562, 2015. 